\title{
Les cités en conteneurs
}

Ou l'habitat dans la logistique globale

The Cities in container or the housing in total logistics

\section{Arnaud Le Marchand}

\section{(2) OpenEdition}

Journals

Édition électronique

URL : https://journals.openedition.org/tc/5889

DOI : $10.4000 /$ tc. 5889

ISBN : 1952-420X

ISSN : 1952-420X

Éditeur

Éditions de l'EHESS

\section{Édition imprimée}

Date de publication : 5 septembre 2011

Pagination : 178-193

ISBN : 978-2-7351-1410-8

ISSN : 0248-6016

Référence électronique

Arnaud Le Marchand, "Les cités en conteneurs », Techniques \& Culture [En ligne], 56 | 2011, mis en ligne le 30 novembre 2011, consulté le 29 septembre 2022. URL : http://journals.openedition.org/tc/ 5889 ; DOI : https://doi.org/10.4000/tc.5889 


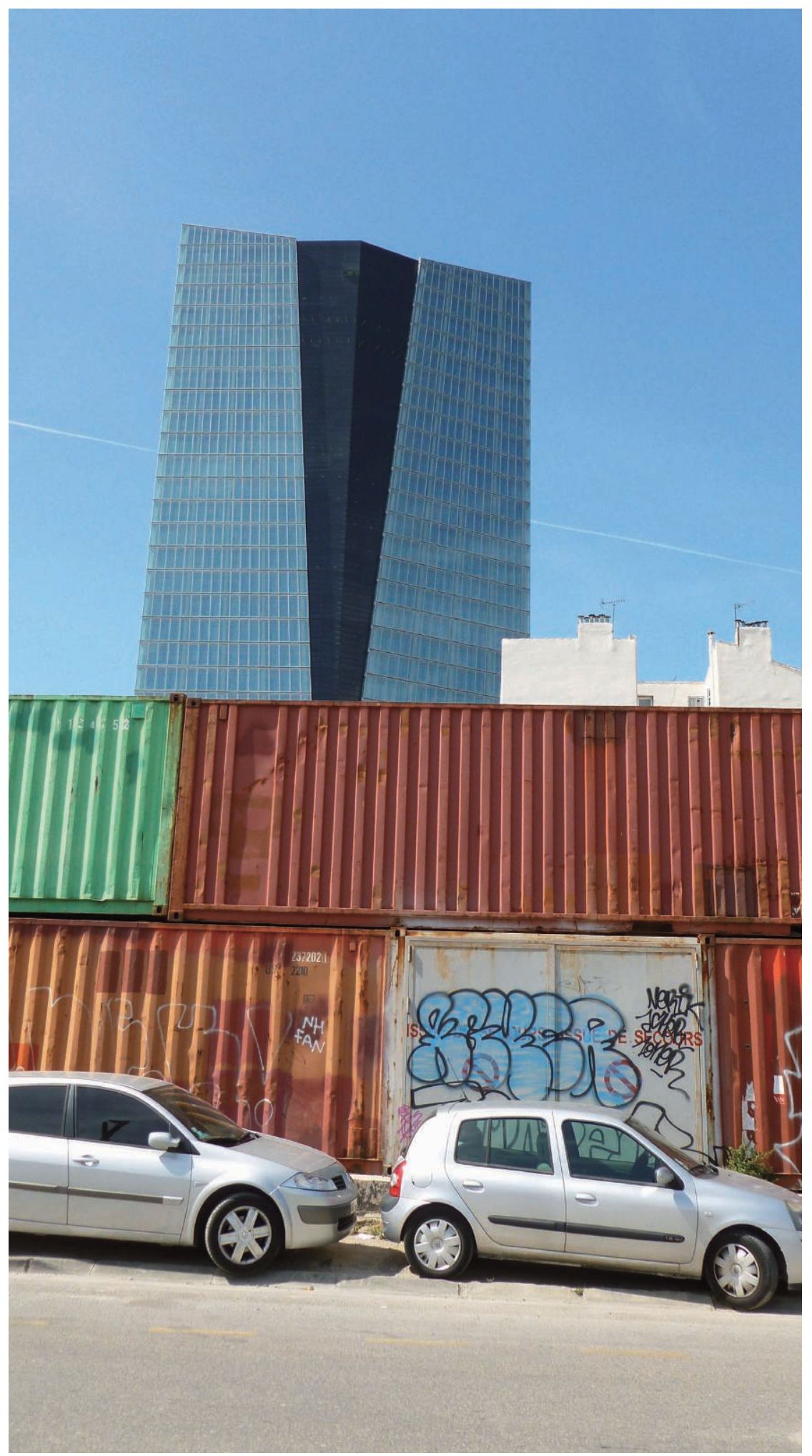

๑ I. Sénépart 


\section{LES CITÉS EN CONTENEURS}

\section{Ou l'habitat dans la logistique globale}

Cette citation, tirée de « La Grande Transformation » de Karl Polanyi lie le commerce maritime et l'habitat, dans un raccourci saisissant, qui fait allusion aux prémices de la révolution industrielle anglaise. À savoir, les enclôts et les transformations de l'agriculture liées à l'exportation de laine, qui eurent comme premières conséquences sociales, de mettre sur les routes, et à disposition du marché du travail, une multitude de paysans pauvres, squatteurs et cottagers, nommés et distingués par leur mode d'habitat (Paul Mantoux 1906). Cette population déterritorialisée allait constituer le patronat et le prolétariat des premières fabriques. Le parallèle avec l'utilisation du conteneur maritime, instrument du transport et support logistique de la mondialisation comme module d'habitation, peut sembler évident, puisque la précarité du travail est réapparue à partir de la fin des années 1970, quand le conteneur commençait à devenir dominant. Les premières utilisations institutionnelles, comme logement, en Allemagne, lui assignait le rôle de cellules de détention pour demandeurs d'asiles et sans-papiers (Wohncontainer). Le but de cet article est néanmoins de nuancer cette impression rapide pour arriver à une autre conclusion. On observera, à cette fin, d'une part que le conteneur habitable n'est pas une réponse mécanique aux changements de conditions de vie depuis la fin du xx ${ }^{e}$ siècle. Il résulte d'innovations par détournement, réalisées très tôt, par des acteurs petits et grands. Les institutions du logement, et de l'architecture, interviennent en bout de processus, en lui accordant une reconnaissance. Cet usage n'est toutefois pas présent partout, alors même que peu de zones du globe échappent à la conteneurisation des marchandises. D'autre part, le conteneur, en tant que mode d'habitat mobile et modulaire, n'est pas entièrement encastré dans l'économie. Polanyi analyse la révolution industrielle comme
«Cette fois encore, l'événement fut particulier à l'Angleterre; cette fois encore, le commerce maritime fut à l'origine d'un mouvement qui affecta le pays tout entier; et cette fois encore ce furent des améliorations menées sur la plus grande échelle qui causèrent des ravages sans précédent dans l'habitation du petit peuple» (Polanyi 1983). 


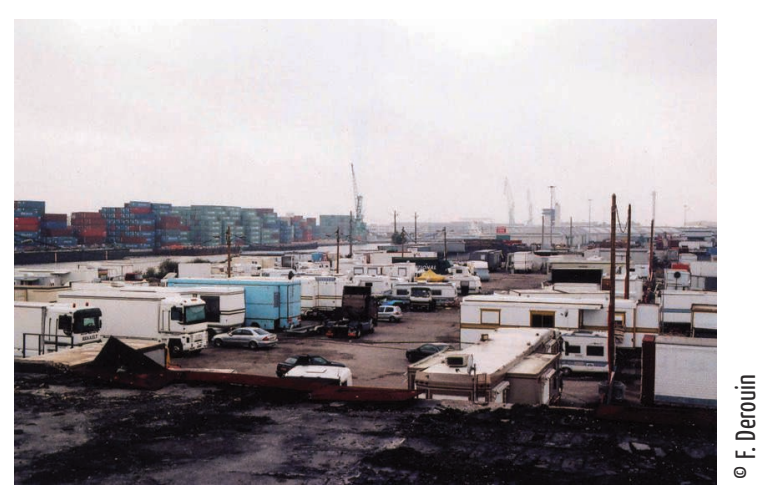

Dépôt de conteneurs et campement forain, à l'occasion de la Foire, dans le port du Havre, 2007.

(Fig. 1) un désencastrement du marché et de la production, qui aboutit à une inversion des valeurs; tous les aspects de la société viennent s'encastrer dans l'économie capitaliste et sont soumis aux impératifs de marchés devenus autonomes. Ainsi, l'économie, sauf à être bornée par l'État, tend à saisir tous les aspects de la vie à commencer par l'habitation, si l'on se réfère à la phrase citée en introduction.

Or, la théorie du désencastrement a pu être remise en cause, par la socio-économie. Selon ce paradigme, l'économie est toujours une construction sociale et politique, elle n'est pas un monde autonome et auto-régulé. L'habitat le plus flexible n'est pas non plus le pur produit de la flexibilité marchande. Pour ce qui nous occupe dans le cadre de cet article, la question se déplace vers les conditions sociales et économiques qui rendent l'habitat en conteneur possible, envisageable et même commercialisable. Par ailleurs, une part d'autonomie par rapport « aux lois du marché » s'exprime dans l'habitat mobile. Si au xix siècle, cet habitat est reconnu comme une nécessité pour les forains et mariniers notamment, dont l'activité ambulante participe de la construction du marché national, il sera considéré comme résiduel, puis réprimé à partir du début du xx $x^{e}$ siècle. La loi de 1912, qui discrimine explicitement les personnes en habitat mobile routier représente l'aboutissement de ce processus. Cependant, ce mode d'habitat a perduré comme résistance contre la sédentarisation, chez des populations et des groupes professionnels marginalisés, ou de façon discrète, non dite, en association avec des activités du travail dans le secteur des transports (Figure 1). C'est notamment le cas des chauffeurs routiers dont une part non négligeable vit dans le camion au moins cinq jours par semaine.

L'habitat non ordinaire mobile (HNO) peut aussi relever du « jeu». C'est le cas dans le secteur du tourisme (tentes, caravanes, etc.). Comme tout jeu, il peut participer d'une « mise en scène », et être temporaire. Il est aussi des occupations " politiques », qui n'excluent pas la dimension ludique ${ }^{1}$. L'habitat mobile s'inscrit ici dans une sorte de jeu, au sens où l'emploie Huizinga (1938), contre les tenants du marché officiel du logement mais aussi contre les normes incorporées dans l'offre et la demande. Dans le cas du logement en conteneur, nous observons une activité de détournement et de recyclage que l'on peut rapprocher de certaines pratiques artistiques.

Avant de s'interroger plus avant sur un possible « changement de qualités » de la boîte et sur le contexte de ces changements, revenons sur la genèse du conteneur maritime et sur les conséquences de son utilisation dans le commerce maritime.

\section{Historique de la « conteneurisation »}

Le conteneur maritime a eu une longue gestation, débutée avant la seconde guerre mondiale. C'est en 1956qu'un transporteur routier américain fonde une compagnie de navigation et désolidarise la caisse des camions pour la charger à bord de navires. La première ligne transatlantique conteneurisée est ouverte en 1966. Deux raisons vont expliquer le succès de cette innovation et sa diffusion générale. D’une part une volonté de réduire les coûts salariaux de manutention, d'autre part le souci de 
rationaliser l'occupation de l'espace à bord des navires (Figure 2). En effet, le transport de marchandises autres que les vracs ou les liquides entraîne un chargement de colis disparates, par conséquent le bateau est souvent « plein » avant d'avoir atteint sa charge maximale. De ce point de vue, le conteneur remet de l'ordre dans le macro système technique de la circulation maritime des marchandises. Mais afin qu'il soit efficace pour le fret international, un travail de normalisation internationale est nécessaire, et une redéfinition des usages de l'outillage portuaire s'impose. On va donc assister à une standardisation des conteneurs, des grues (qui deviennent des portiques), des navires et des espaces portuaires. À partir de 1974, les acteurs du monde maritime considèrent que le système technique est au point (Fiore 1982, Le Marchand 1997). Le conteneur est devenu un objet normé et maîtrisé. Il va commencer à produire ses effets. Il sera le support technique de l'accroissement des échanges internationaux de marchandises et permettra une production modulaire de biens complexes (comme les automobiles), sur plusieurs sites dispersés sur plusieurs continents. De ce point de vue, il est aussi important que la finance et l'informatique dans le processus de mondialisation. Il a aussi un impact culturel sur la vie portuaire et les représentations du commerce international. En effet, le commerce de marchandises manufacturées, appelées les " marchandises diverses ", au sens d'inclassables, par opposition aux matières premières transportées en vrac sur des navires spécialisés (pétroliers, minéraliers, etc.), était encore vu comme un trafic résiduel en regard de l'approvisionnement des industries lourdes. Avec la conteneurisation, la logistique va pouvoir s'appliquer aux produits finis. Or à partir de la fin des années 1970, le commerce de ces produits devient le vecteur du développement des échanges (Krugman 2006). Il s'agit notamment pour les pays industrialisés d'accroître les exportations pour payer la facture pétrolière, et ensuite d'acheminer les biens dont la production a été délocalisée. De résiduelles, ces activités deviennent cruciales et croissantes. Or, pour mesurer ces échanges, on ne peut utiliser seulement le poids, comme on le faisait pour les vracs. Il n'y a pas non plus de mesures monétaires, pour des raisons comptables. C'est alors le nombre de conteneurs (E.V.P, équivalents vingt pieds) chargés et déchargés qui devient l'unité mondiale de compte des activités portuaires. Sur toutes les places portuaires le design des quais et de l'outillage portuaire se conforment aux standards de la circulation des conteneurs. On parlera alors de flux de conteneurs, bien qu'il ne s'agisse pas de fluides, comme les hydrocarbures ou les grains (LeroiGourhan 1971). Le trafic est effectué conteneur par conteneur, boîte par boîte, la chaîne de manutention est discontinue. Chaque boîte est identifiée et nécessite des soins particuliers. La réparation de conteneurs devient très vite une nouvelle activité dans les zones portuaires. C'est de cette activité que naissent les aménagements spécifiques pour « usages industriels » et que se développeront les compétences nécessaires à l'utilisation du conteneur comme module d'habitat. Sous cet angle généalogique l'origine routière du conteneur peut rapprocher cette transformation de celles qui ont abouti à la roulotte foraine et au camping-car à partir du chariot et du

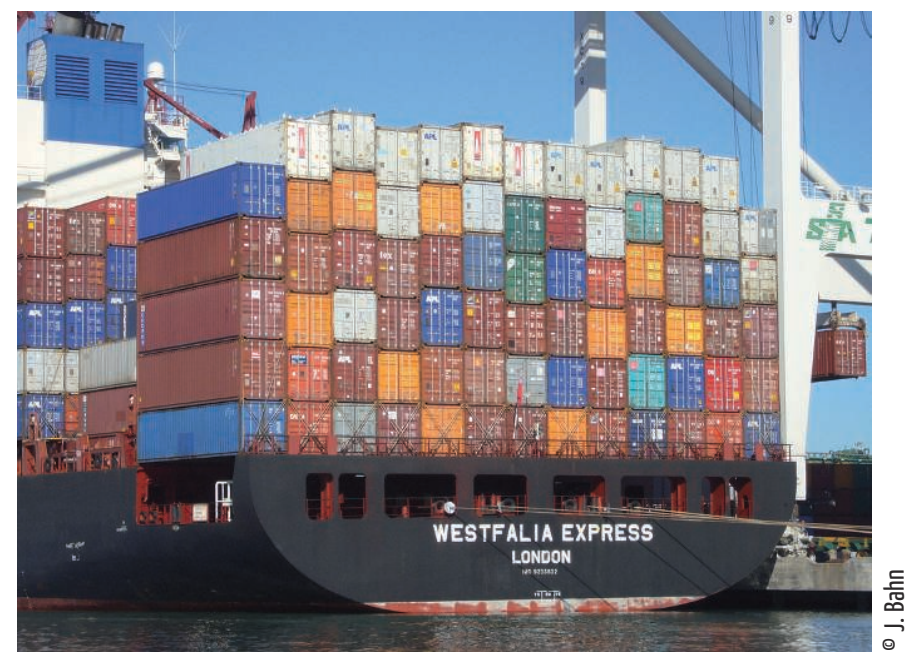


camion (Wallis 1997). L'objet conteneur virtuellement en devenir trouve un nouveau domaine d'application au sein de systèmes techniques dont les acteurs ne sont plus des activités logistiques ni des marins, ni des marchands ambulants.

\section{Outil dans un nouveau contexte urbain ?}

Si le conteneur fut l'un des supports physique et technique de l'accroissement des échanges, il semble prendre un autre rôle dans la nouvelle géographie économique qui a suivi cette mutation du commerce international. La mondialisation économique du xxI ${ }^{\mathrm{e}}$ siècle est, par opposition au commerce entre Nations du $\mathrm{xIx}^{\mathrm{e}}$ et du $\mathrm{xx}^{\mathrm{e}}$ siècle, une internationalisation de la production. Si l'on suit les analyses d'Albert Michallet (2004), le changement réside dans la mobilité des facteurs de production, qui étaient relativement fixes dans le paradigme de l'économie internationale (où ce sont seulement les produits qui circulent). Ce sont les fameuses délocalisations qui accompagnent un flot croissant d'investissement direct international. Or, ce mouvement est synchrone d'un développement du commerce intrabranches: des échanges croissant au sein du même secteur ${ }^{2}$, ce n'est plus seulement un échange de produits industriels contre des matières premières, par exemple. La géographie économique s'en trouve changée, elle apparaît comme plus intriquée (Krugman 1998). Ce qui se traduit aussi par le sentiment qu'il y a maintenant du « Nord dans le Sud » et du «Sud dans le Nord ». L'organisation même de la production industrielle est maintenant « multi-site » plutôt qu'à la recherche de production sur un seul site. La tendance va aller dans le sens d'un découpage du produit final en modules réalisables dans différentes parties du monde, selon les compétences localisées et réassemblées au dernier moment. Ces phénomènes créent une répartition fractale de la production qui génère une intrication des activités à toutes les échelles d'analyse. Cet aspect fractal résulte de la complexité des processus, alors que la planification urbaine et industrielle a perdu beaucoup de terrain, au profit d'interventions au coup par coup (Graham \& Marvin 2001). Cette répartition est en outre plus changeante que par le passé. Elle a pour conséquence la création et la disparition concomitantes d'interstices, de friches, urbaines, industrielles, touristiques, etc. Les deux mouvements s'auto-entretiennent. Ils aboutissent à des occupations temporaires de l'espace, qui peuvent être informelles ou institutionnelles. Dans ce cas, on devrait parler d'occupations transitoires de l'espace ; il peut s'agir par exemple d'occuper une friche pour permettre une requalification contrôlée par les propriétaires ou les autorités municipales. À une autre échelle, parce qu'elle se déploie sur plusieurs sites, la fragmentation des processus productifs s'effectue dans une intégration internationale des villes et des territoires. Les interstices se développent sous l'effet de l'intrication des espaces productifs et urbains, rendue nécessaire par l'utilisation de compétences et de services localisés dans les villes. Comme ce redéploiement est mouvant, friches et enclaves réapparaissent sans cesse.

C'est dans ce contexte, dont il est l'un des éléments, que le conteneur habitable fait son apparition officielle et médiatique. Il s'agit bien sûr du projet «Container City à Londres » en 2001, pour requalifier une friche portuaire entre Docklands et Canning Town (Le Marchand 2007), des cités pour étudiants en conteneur en Hollande (Amsterdam 2005) et en France (Le Havre 2010). Chaque fois, l'intention du projet est reliée à cette occupation transitoire, a priori l'installation est démontable et n'a pas vocation à rester à demeure. Dans les différents cas, il s'agit de zones proches des anciens sites industriels et portuaires, 
voire d'anciens dépôts de conteneurs. Dans ces zones l'usage du « conteneur bureau » ou « atelier mobile » a précédé de plusieurs années l'installation de ces équipements. Il est tentant de relier cette innovation à la logistique globale. D'autant que dans d'autres pays (Chine et Corée), les conteneurs maritimes aménagés sont des modes d'hébergement des travailleurs sur les chantiers, eux aussi connus depuis plusieurs années. Ces usages préexistants, peut-être dès l'apparition de ces contenants, contribuent très certainement à expliquer leur adoption comme logement pour étudiants aux Pays-Bas et plus généralement dans des cités portuaires. Enfin, les dépôts de conteneurs voisinent déjà souvent avec d'autres habitations mobiles: campements d'industriels forains, d'ouvriers sur des chantiers industrialoportuaires (Figures 1 et 3), d'artisans itinérants ou de migrants clandestins. Selon cette première lecture, le conteneur habitable apparaît bien comme la traduction de pratiques issues de la production mondialisée, en voie d'extension à de nouveaux aspects de la vie. Ceci nous ramènerait à la citation de Polanyi mentionnée au tout début de cet article. Enfin, comme ces cités en conteneurs servent clairement de têtes de pont au « renouvellement urbain », voire à une recherche de gentrification, puisqu'étudiants et artistes sont des nouveaux venus dans ces quartiers ouvriers, il est tentant d'achever la critique par le dévoilement d'une utilisation «foncière » de ces dispositifs. En envisageant ces containers comme un moyen de faire monter les prix du terrain dans des zones en requalification.

\section{Le cas du Havre et les réticences d'une partie de la population}

En tant qu'économiste, nous avons participé aux travaux préparatoires à l'installation de logements en conteneurs au Havre, nous avons réalisé des entretiens dans la communauté universitaire et dans la ville et ceci nous a permis de voir apparaître des réticences autres que celles qui relèvent d'une critique du Capitalisme. En effet, ces projets ont fait l'objet de plusieurs critiques, qui indiquent un conflit de représentations. Les conteneurs aménagés pour étudiants au Havre, malgré l'augmentation de la surface du logement $\left(25 \mathrm{~m}^{2}\right.$ contre 14 ou 9 , pour les chambres «normales»), ont été parfois rejetés. Des arguments différents ont été avancés.

Certains de mes interlocuteurs (membres de la communauté universitaire, élus locaux) ont dénoncé une assimilation des étudiants aux marchandises. Le conteneur est vu avant tout comme un support du commerce, dont le recyclage n'efface pas la destination première. Ce qui entre à l'intérieur devient une marchandise. Cette crainte s'est notamment traduite par l'expression « les étudiants en

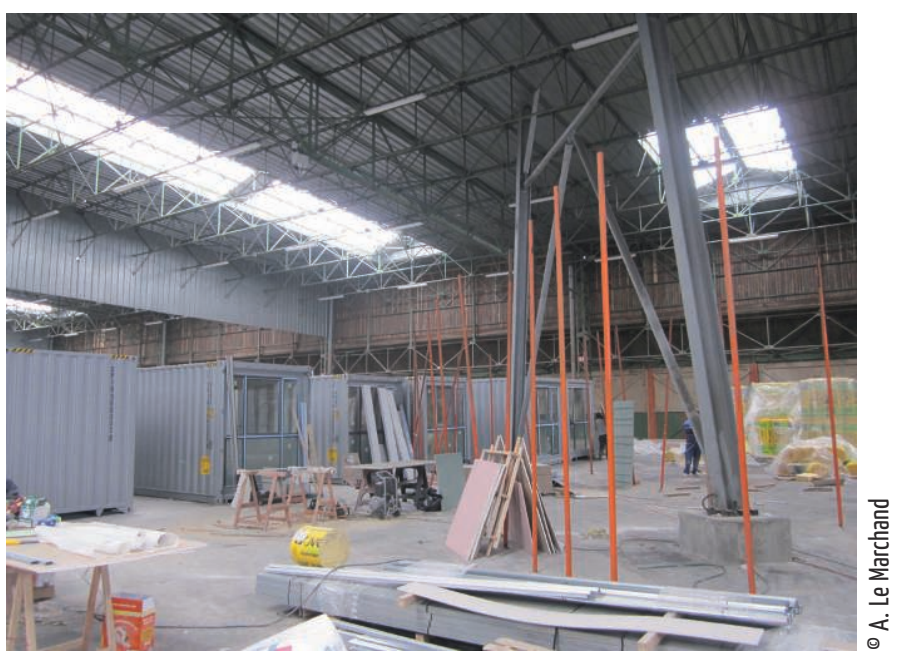

Chantier industriel d'assemblage d'équipements modulaires pour l'industrie gazière, avec campingcars. Port du Havre été, 2009.

(Fig. 3)
Chantier de préparation des conteneurs pour la cité universitaire, dans un hangar portuaire du Havre, été 2010.

(Fig. 4) 
boîte », reprise à titre ironique dans une manifestation contre la Loi sur les Responsabilités de l'Université (LRU) au Havre puis dans le titre d'un article de presse (Le Havre - Presse 23 janvier 2009).

Parmi les habitants du Havre, quelques-uns ont fait part d'une aversion envers un dispositif qui semble incarner le déracinement. L'absence de fondations, la possibilité de retirer rapidement (au moins théoriquement) les conteneurs sont envisagés comme un symbole de la perte d'ancrage territorial. Le directeur d'une entreprise de réparation et d'aménagement industriel de conteneurs, nous a évoqué des oppositions similaires à un projet de base-vie pour salariés en déplacement à l'étranger. Plusieurs personnes interrogées redoutent « d'habiter le voyage » (Bouillon 2009), et rejettent ce type de constructions modulaires et mobiles.

Enfin, des universitaires (juristes) ont émis lors de discussions sur le projet, des réserves d'un autre ordre. Puisque ces logements n'ont pas de fondations, ils les assimilent à de l'habitat précaire et indigne. Ils soulèvent des interrogations sur les droits des personnes ainsi logées. Ils redoutent une extension du statut juridique précaire des habitants de logements mobiles et craignent par exemple que les étudiants ainsi logés n’aient pas le droit de vote, dans la mesure où cet habitat pourrait être assimilé à du camping ${ }^{3}$. Ces universitaires recommandent de bien s'assurer que les habitants aient des droits clairs. Le souci est donc inverse de ce qui a pu être observé pour les terrains de camping, où le flou des droits est la règle (Raveneau \& Sirost 2001). Se pose aussi la question des droits à l'aide au logement pour les étudiants. Cette interprétation correspond à la crainte du déclassement, et de l'assimilation des étudiants à des travailleurs immigrés, à des marchands forains, ou à des Tsiganes. Ici apparaît un problème de convention, de conflit entre les adaptations de la politique de logement à une nouvelle donne urbaine et d'autres référents républicains plus traditionnels.

Nous allons à présent considérer d'autres phénomènes qui nous permettront de nuancer voire de contredire les conclusions polanyiennes que nous pourrions tirer de ces premières observations.

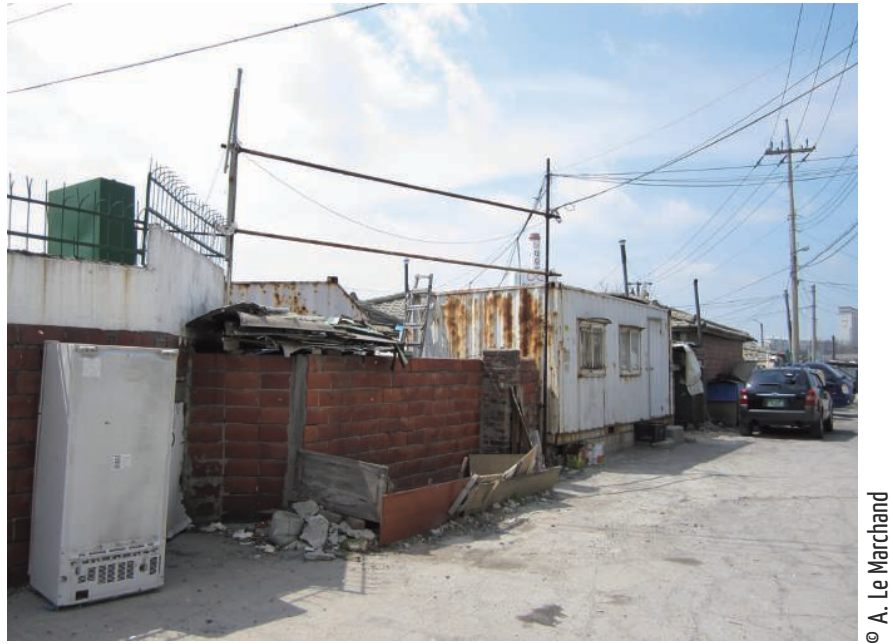

Habitat pauvre dans les interstices de la ville portuaire d'Incheon, Corée du Sud avril 2010. (Fig. 5a)

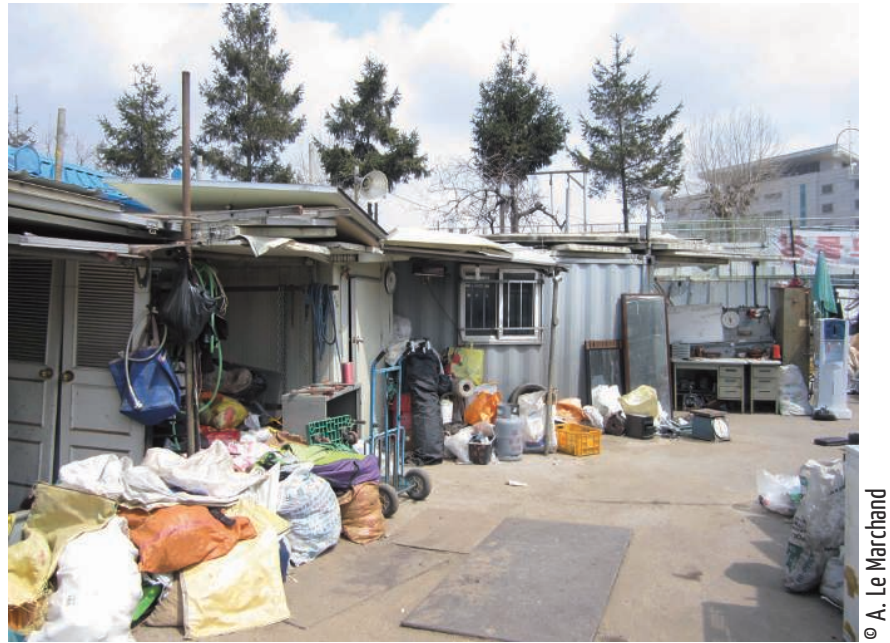

Conteneurs intégrés à l'économie de recyclage et de bazar, Incheon, Corée du Sud avril 2010. (Fig. 5b) 


\section{Le recyclage du conteneur comme ruse et résistance}

Pour commencer, nous observerons que l'intrication entre diverses fonctions sur un même espace n'implique pas forcément une domination par « l'économique » des autres aspects de la vie sociale. À la limite, et à propos des villes portuaires, l'intrication était la règle quand les entrepôts du vieux négoce étaient également l'habitat du négociant, voire de ses salariés et ce avant que les activités portuaires ne soient physiquement désencastrées de la cité (Bonillo \& al. 1992). C'est-à-dire avant que la ville industrielle et la tendance à la séparation fonctionnelle de l'espace urbain ne se développent. Le retour de l'habitat intriqué ou proche des espaces portuaires peut ainsi être lu comme une réintégration de la ville, et non seulement comme un processus de fragmentation économique.

Par ailleurs, l'usage des conteneurs comme habitat vient d'acteurs non institutionnels et souvent situés hors entreprise. De nombreux témoignages signalent par exemple la présence de « conteneurs logements » en Afrique, dès le début de la conteneurisation. Lors d'une enquête en 2007 sur les logements des salariés en déplacement, j’ai rencontré des Manouches vendeurs de caravanes qui avaient construit, dans la banlieue parisienne, une maison en conteneur camouflée avec du bois et ce dès les années 1990. L'usage du conteneur comme atelier fut le fait de petites entreprises, assez éloignées des grands circuits de la production mondialisée, voire du monde rural. La première mention du conteneur habitable dans un rapport officiel sur l'activité maritime apparaît en 2003. Il y est fait mention de la découverte fortuite d'un clandestin dans un navire escalant au Hub De Giaoa Tauro, en Calabre en 2001. Cet homme projetait un attentat aux USA, et était caché dans un conteneur dont l'aménagement avait surpris l'équipage (Comité des Transports Maritimes de l'OCDE 2003).

Deux séjours dans la métropole séoulitaine en 2007 et 2010 m’ont permis de mener une petite enquête sur place. En Corée du Sud, le conteneur (Figures 5a à 5d) est très

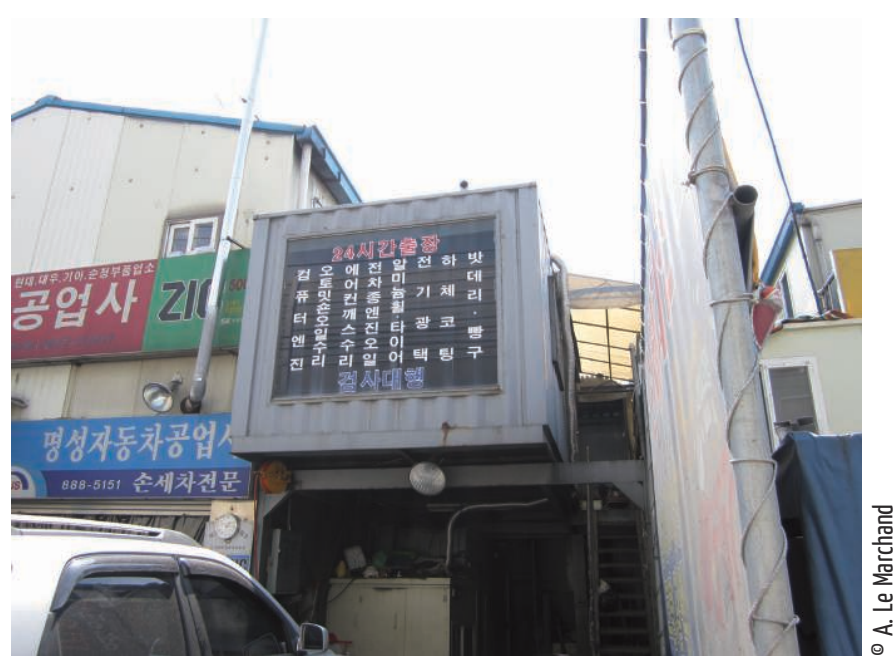

Dans l'architecture des petites entreprises, un garage à Incheon, Corée du Sud avril 2010. (Fig. 5c)

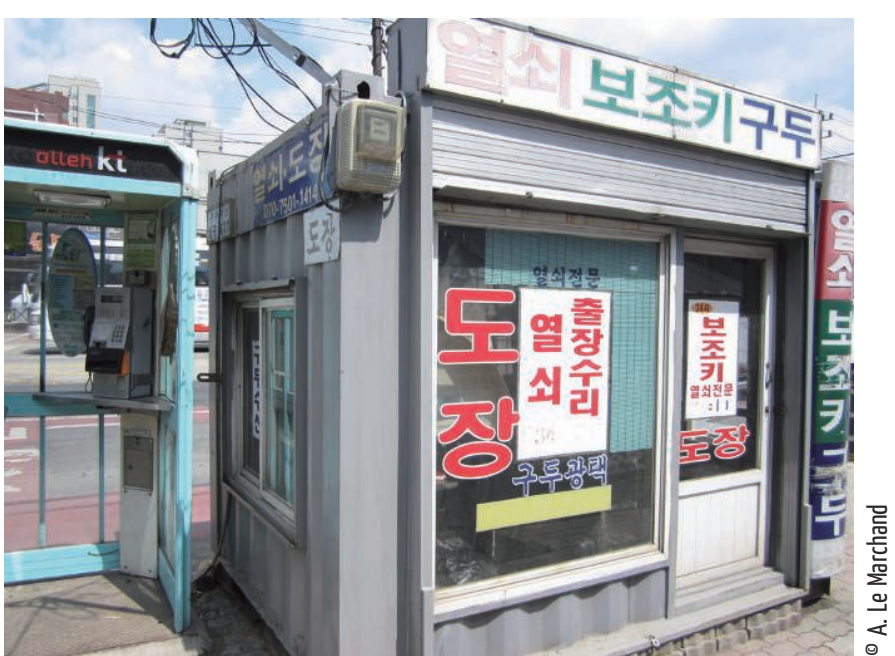

Des boutiques dans les rues d’Incheon, débouché portuaire de Séoul. Corée du Sud avril 2010. (Fig. 5d) 
présent dans les secteurs informels du logement et du recyclage où il sert d'entrepôt et de magasin. Il est utilisé comme échoppe dans le petit commerce de rue. Son changement de fonction s'est opéré dans les marges de la société coréenne. Le responsable d'une agence économique française en Corée, explique notamment qu'il existe une surtaxe au transit de conteneurs vers la Corée du Nord. La pratique, suffisamment courante pour justifier une provision systématique, consiste à détourner une «boîte » pour en faire un logement et donc à ne pas la renvoyer après la livraison. Il s'agit de procédés camouflés ou « clandestins », officiellement considérés comme résiduels. Ils changent de statut à la faveur de la crise. En effet quand ce type d'habitat disparaît le nombre de sans-abris augmente. C'est le cas dans le centre de Séoul où depuis la crise de 1997, ce phénomène est amplifié par la politique de renouvellement urbain qui substitue des constructions de prestige à l'ancien habitat bricolé, alors que le travail précaire et le chômage augmentent. Comme il reste des zones de travail « irrégulier » au cœur de la métropole, des travailleurs pauvres doivent vivre dans des secteurs où les loyers sont trop élevés pour leurs revenus. Dans ce cas, le carton remplace le conteneur. L'habitat constitué d'objets recyclés n'est donc pas en voie de disparition, ce qui peut inciter à reconsidérer le statut du conteneur habitable.
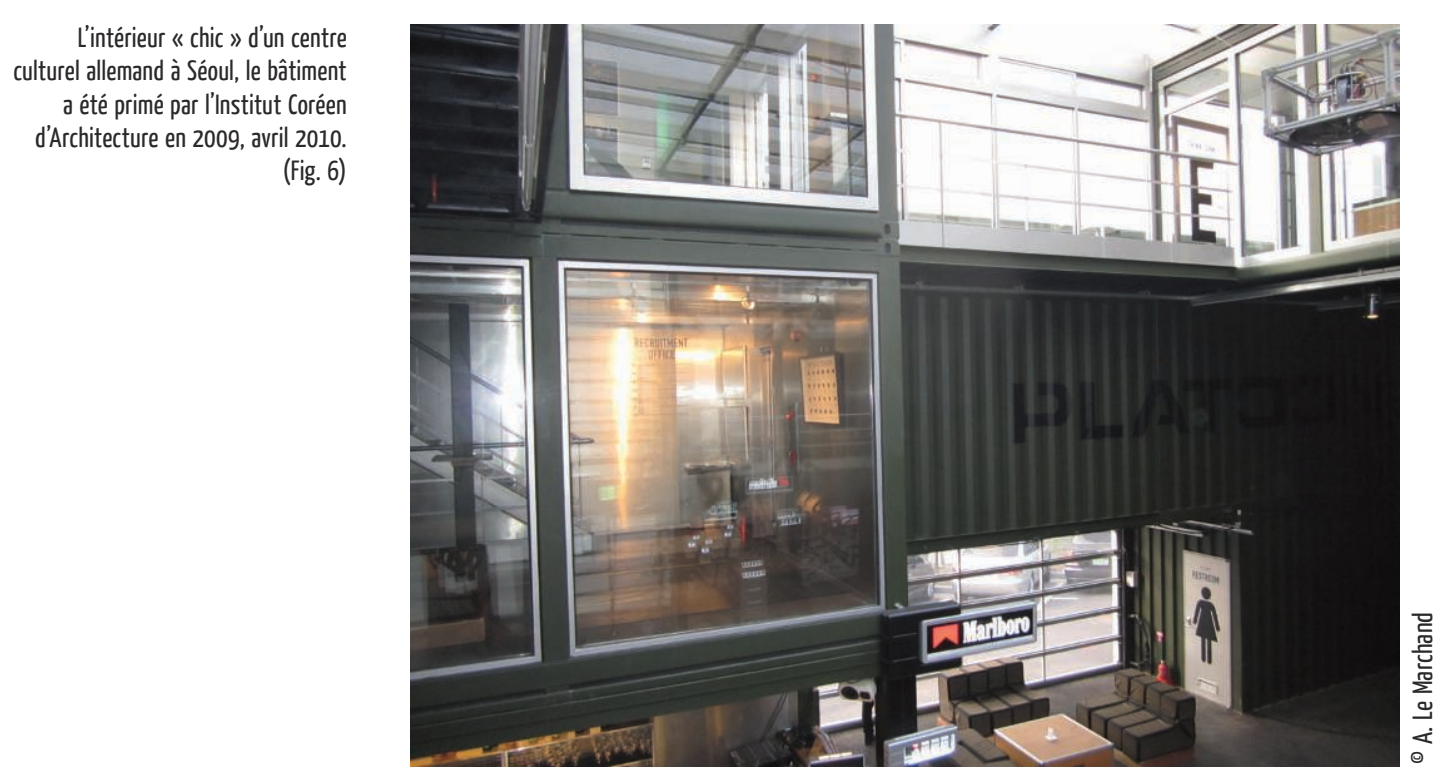

Ainsi, il existe à Séoul même, depuis 2009, un équipement culturel allemand (Platoon Kunsthalle - Figure 6), réalisé en conteneurs maritimes. Le chauffeur de taxi qui nous y amena, pensait que le chantier ne devait pas être terminé et qu'il s'agissait du logement des ouvriers. Mais il s'agissait bien d'une récupération par une architecture officielle. Ce bâtiment a par ailleurs reçu un prix national, « architecture award of Korea », décerné par l'institut Coréen d'architecture en 2009. C'est la plus haute distinction coréenne dans ce domaine. C'est une phase de la reconnaissance du caractère fonctionnel et légitime de ce type d'habitat 
pratiqué, à l'origine, par les « outsiders » de la société coréenne. Le conteneur y est d'abord détourné par les salariés et les travailleurs non intégrés à la grande entreprise, ce n'est pas une production des entreprises et des acteurs insérés dans le commerce international, mais il conquiert à présent une place culturellement légitimée. Son utilisation comme logement indique que des espaces, même apparemment marginaux, sont connectés à la circulation des marchandises. Leur transformation n'est pas pour autant automatique, ce sont les acteurs qui ont adapté les conteneurs. On peut généraliser cette remarque à des pratiques, pourtant explicitement associées à des discours de ruptures avec le salariat et la consommation. Ainsi, lors d'un rassemblement organisé en France par Halem, l'association de défense des habitants en logements éphémères et mobiles, journées dénommées « Agora de l'habitat choisi », à Bussière-Boffy en juillet 2009, les yourtes sont présentées comme exemple d'éléments d'un mode de vie revendiquant une perspective « décroissantiste ». Durant les discussions, formelles et informelles, plusieurs participants admettront que nombre de tentes sont réalisées avec du coton chinois, parfois issu d'organismes génétiquement modifiés, même si d'autres utilisent du coton bio (probablement transporté en conteneurs). Ainsi il n'y a pas de rupture totale entre la production de ces habitats et les conditions matérielles de production prévalant dans le système global. De ce point de vue, le conteneur logement n'est pas plus symptomatique de la mondialisation que d'autres dispositifs, même s'il peut en être une métaphore. Une opération de recyclage peut très bien être un retournement de l'objet contre son système d'apparition.

\section{L'usage des modules peut être une résistance ou une ruse}

L'habitat mobile résulte des « arts de faire », tels que les a présentés Michel de Certeau (1990). Du point de vue d'une théorie économique institutionnaliste, il rappelle que face à un marché normé qui les exclue, certains acteurs peuvent «bricoler » avec les ressources matérielles, voire juridiques, pour aménager des espaces et des produits davantage conformes à leurs attentes. L'institution ne recouvre pas tout le champ des possibles, dans ce cas une production peut, aussi, être une résistance au « lit de Procuste » des conventions dominantes. L'histoire de l'habitat modulaire et mobile est prise dans une critique du fonctionnement du marché « immobilier », ici le terme prend un sens très physique. Ensuite, on assiste à une reconnaissance par l'institution de la récupération du conteneur pour cet usage non prévu à l'origine, prélude à son réencastrement.

\section{Les réponses des institutions}

Qu'en est-il de ces constructions légitimées par les institutions (logements universitaires, centre culturel, ensembles privés) ? Ces projets « institutionnels » peuvent-ils aider à légitimer ces formes d'habitat et rejoindre ou soutenir des demandes de droits, émanant d'acteurs moins « qualifiés » juridiquement ? On serait tenté de répondre par l'affirmative, mais l'examen du cas havrais incite à nuancer ce point de vue. Il semble même que les institutions cherchent à éviter cela. Lors d'une des réunions préparatoires au projet d'installation de logements étudiants à base de conteneurs à laquelle nous avons assisté, une des parties prenantes précisa qu'il ne s'agissait pas de « construire un camp de nomades » mais d'acquérir des compétences sur le conteneur et de travailler à la requalification du 


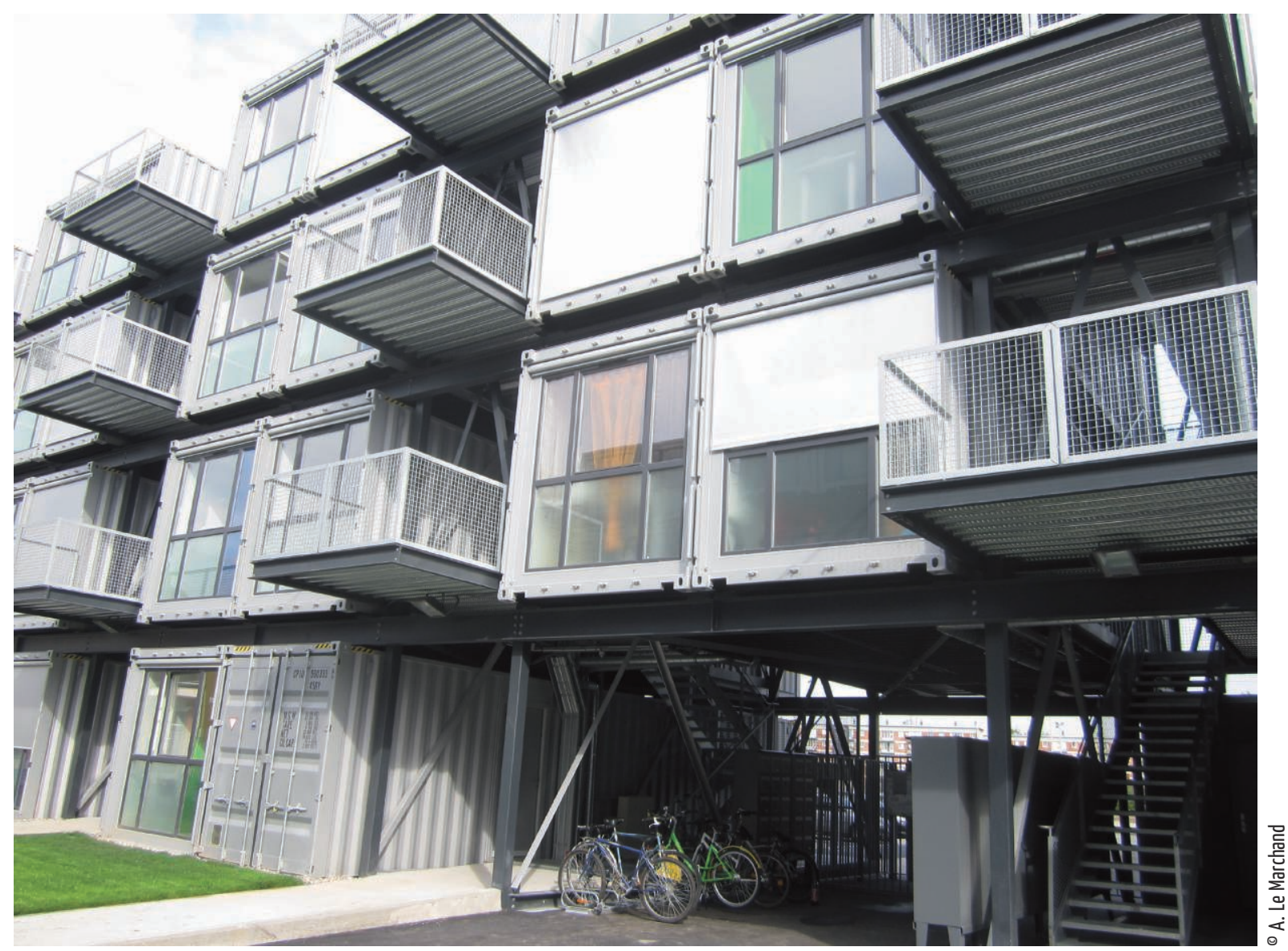

\section{Cité Universitaire au Havre, rentrée 2010}

Les conteneurs sont posés dans une structure métallique et non pas empilés.

Les précautions pour éviter l'assimilation à l'habitat mobile ne sont donc peut-être que des ruses, des contournements, eux aussi transitoires. Dans ce cas, deux évolutions différentes se dessinent:

- la première irait dans le sens d'une normalisation générale de l'habitat modulaire mobile, type conteneurs ou autres. Cette hypothèse serait compatible avec l'idée d'un habitat mobile en voie d'intégration dans une économie valorisant la flexibilité et les usages temporaires de l'espace. Dans ce cas, on pourrait sans doute retracer une diffusion à partir des villes portuaires ;

- la seconde verrait se développer une segmentation en fonction des habitants. Ce ne sont pas les caractéristiques techniques de l'habitat qui le rendent légitime, mais ses habitants. Ainsi l'habitat en conteneur sera-t-il reconnu éligible aux aides sociales aux logements pour les étudiants, mais pas pour d'autres personnes. Pour le moment, c'est cette voie qui semble la plus probable. Mais elle peut aussi déboucher sur des remises en cause, si la discrimination devient trop voyante ou trop contradictoire vis-à-vis d'autres normes juridiques ou d'usages informels. (Fig. 7) 
quartier. S'agissant du droit à l'aide au logement pour les étudiants, on contourna l'objection de précarité, l'Aide Personnalisée au Logement (APL) exclut de son champ d'application les immeubles qui n'ont pas «normalement » de fondations réelles, via une qualité sociale provenant du mode de financement. Comme la cité universitaire est financée par des Prêts Locatifs Sociaux (PLS), ses occupants seraient de droit, éligibles à l'APL et évitant ainsi une jurisprudence étendant la généralisation de l'APL à des habitats légers ou mobiles. Un des acteurs de l'aménagement des conteneurs, parlera à ce propos, de « soutien politique ». Cette attitude prudente se traduit aussi dans les choix techniques. À la différence des réalisations anglaises et hollandaises, au Havre, les conteneurs ne sont pas empilés directement, mais posés dans une structure métallique (Figure 7). Cette disposition répond aux normes techniques françaises; il s'agit en cas d'incendie, d'éviter la propagation du feu, laquelle serait capable de provoquer un effondrement de l'ensemble ${ }^{4}$. Cette solution permet par ailleurs de déclarer des fondations (ce qui est un peu discutable car les conteneurs peuvent être retirés de cette structure. Ils n'ont pas vraiment d'emprise au sol). L'enjeu ici est aussi d'éviter le qualificatif d'habitat mobile. Il est pourtant prévu, par la municipalité et l'Université, de déplacer l'ensemble dans quelques années, une fois la requalification du quartier terminée. Les acteurs impliqués dans ce projet espèrent également pouvoir mettre en œuvre toutes les possibilités du conteneur, c'est-à-dire l'empilement direct, sans structure supplémentaire, à l'occasion d'un projet ultérieur. Cette réalisation est donc perçue, par les opérateurs, comme un premier pas, un test, pour aller vers des constructions plus souples à l'instar des cités hollandaises. Une des entreprises impliquées (ARC) dans la construction de la cité universitaire havraise développe notamment un projet qui ajoute une nouvelle qualité au logement en conteneur. À l'occasion du tremblement de terre en Haiti, le 12 janvier 2010, on s'est rendu compte que les boîtes sur le port avaient résisté aux secousses parce qu'il s'agit de parallélépipèdes fermés et relativement souples. Le conteneur maritime a été conçu pour résister à des accélérations lors des manutentions par les portiques et aux mouvements imprimés par la houle aux grands navires. De fait, il correspond aux normes antisismiques. Pour la reconstruction d'Haiti, une association (regroupant certains techniciens de l'entreprise de réparation de conteneur et d'autres personnes) a mis au point un conteneur habitable avec énergie solaire, système pour récupérer l'eau de pluie et fournir de l'ombre. Le conteneur pourrait en outre être expédié en transportant 21 tonnes de produits. Il serait donc habitable, auto-suffisant, toujours utilisable comme moyen de transport et antisismique ${ }^{5}$. L'évolution de l'objet n'est pas terminée et ne s'oriente pas vers un abandon de la mobilité.

\section{Du standard à l'objet singulier}

Lultime question que nous souhaitons soulever dans cet article consiste à comprendre comment le conteneur habitable peut s'intégrer dans une représentation plus large. Si nous utilisons le modèle de « cité » développé par Boltanski et Chiappello (1999) ${ }^{6}$, nous pouvons considérer que les qualités des constructions en conteneurs sont défendues dans le cadre d'un discours « connexionniste », caractéristique de l'émergence d'une « cité par projet $»$ qui valorise les liens avec les activités portuaires, l'attraction d'étudiants, voire celle de la « classe créative » (pour Container City à Londres). En outre, prédomine la gestion par projet; ce ne sont pas des constructions prises dans un plan d'ensemble, il y a une dimension opportuniste. Ces dispositifs semblent bien relever d'un « Nouvel Esprit 
du Capitalisme » (ibid.), qui valorise les réseaux, la variété des produits et les réalisations ponctuelles. Cette intégration passe enfin par un passage de l'économie du standard à celle des « biens singuliers » (Karpik 2007). Le conteneur destiné au départ à un usage indifférencié de masse devient un bien adapté à des contextes et à des usages précis.

Cependant, en tant qu'habitat dont l'histoire a commencé avant celle des réalisations officielles et médiatisées, il s'inscrit aussi dans un héritage critique et moderniste de l'urbanisme (Kopp 1978). On peut le rattacher à des pensées antérieures de la ville. Ainsi, Hector Horeau qui fut l'un des précurseurs de la construction métallique, écrivait en 1868 dans « l'Édilité urbaine », dans une énumération de projets pour réformer les villes: « Dans quelques cas, faire des constructions démontables, portatives ou roulantes, sur les terrains à vendre et qui par la rapacité des possédants restent très longtemps inoccupées ». Il s'agit donc dans ce cas d'un dispositif contre la spéculation. Hector Horeau sera l'architecte nommé par la Commune de Paris en 1871. Le type d'habitat qu'il évoque ici trouvera des prolongements dans les « milieux libres ». Il sera porté par un courant nomadiste, et des personnes recherchant des modes de vies alternatives ${ }^{7}$; il sera le support de fuite hors du salariat autant que vers le monde forain au début du $x^{e}$ siècle. On peut considérer que les projets « officiels", tels ceux présentés dans cet article, sont des entreprises de récupération de ces dispositifs, qui en changent la signification. Ce n'est dès lors pas seulement l'objet conteneur qui est recyclé et détourné, mais aussi les rêves et idéaux qui l'environnent. Il y a bien néanmoins une ouverture vers une reconnaissance de la fonctionnalité urbaine de l'habitat modulaire et mobile. Ainsi, les racines théoriques de l'habitat en conteneur sont autant à chercher du coté de préoccupations sociales, que des pratiques spéculatives.

Ainsi, adaptation et contestation en actes peuvent se combiner autour d'un même objet détourné. La précarité génère l'invention de solutions qui peuvent « monter en généralité » et trouver une légitimité culturelle et politique, autant que simplement économique. Nous soulignerons pour finir l'ultime paradoxe qui réside dans la singularisation du conteneur opéré par cette transformation. Comme moyen de conditionnement et de transport, la boîte est standard; comme habitation, elle devient singulière au sens de «l'économie des singularités » (Karpik 2007). Cette utilisation fait débat et suscite des incertitudes sur le plan du confort (des interrogations sur l'isolation reviennent souvent); sur le plan du statut juridique de ses occupants, sur la construction adéquate et... sur la mobilité réelle de ces dispositifs, puisque pour l'heure, aucune de ces « cités » n'a encore été déplacée. La différence et la singularité tiennent aussi à l'architecture, les cités sont « mises en scène », alors que rien ne ressemble plus à un dépôt de conteneurs qu'un autre dépôt de conteneurs. La singularisation est un produit du recyclage. Notons au passage que presqu'un an après l'ouverture de la cité du Havre des défauts sont apparus, notamment des fuites et une répercussion des vibrations engendrées par le passage des camions sur la chaussée. Néanmoins le CLOUS du Havre (Centre Local des Oeuvres Universitaires et Scolaires) annonce (Le Havre Presse du 25 mai 2010), que 42 \% des résidants ont décidé d'y rester, la moyenne étant d'un tiers pour les autres cités construites de façon conventionnelle (le logement universitaire est a priori un logement de passage). Il n'est finalement pas très étonnant que l'habitat mobile soit concerné par la logistique globale. Les points de contact avec le macro-système technique de la circulation des marchandises étant nombreux, il était prévisible qu'une influence s'exercerait. Il est plus intéressant en revanche de comprendre comment les usages, feront en retour évoluer l'objet conteneur. Ce ne sont dès lors pas les figures de l'encastrement ou de l'exclusion qui nous permettent de penser 
ce type d'habitat mais l'enchevêtrement et les dynamiques d'innovation. Les données classiques du marché du logement, comme le prix, expliquent en partie les évolutions. Mais nous nous trouvons au-delà d'une simple réponse mécanique, d'inventivité et de valeurs. Le conteneur habité participe d'une longue tradition de la mobilité, nationale et trans-nationale, qui cherche une reconnaissance pour sortir de la furtivité.

\section{NOTES}

Photo d'ouverture : Exemple du détournement du conteneur dans un espace de friches urbaines en devenir dans le quartier d'Arenc à Marseille, 2011. En premier plan, le batiment des « Docks des Suds » espace populaire dédié à la culture, constitué en grande partie par l'aménagement de conteneurs récupérés sur l'espace portuaire proche. En arrière plan la tour hébergeant le siège de la société CMA-CGM, troisième transporteur maritime mondial.

1. Notons par exemple que certains militants de l'association « l'Intersquat», qui a pour objectif de relier les squatteurs, sont aussi des organisateurs de jeux de rôle dans les locaux occupés.

2. La France, par exemple, importe et exporte des automobiles, des produits agricoles, etc.

3. À propos du flou ayant cours dans les terrains de camping: Sirost 2001 et Sirost, Raveneau 2001.

4. Les « techniciens » de l'entreprise réalisant les aménagements pensent que cette solution, qui alourdit le coût du projet, n'était pas incontournable. On aurait pu, selon eux, démontrer la compatibilité de l'empilement avec des normes de sécurité françaises, mais au prix d'un délai supplémentaire dans la préparation du dossier. Délai qui aurait compromis les chances du projet d'être éligible pour certains crédits liés au plan de relance de l'économie.

5. Cette tentative n'a pas abouti pour le moment. D'autres projets similaires existent, par exemple l'association Ede Timoun Yo a expédié dix conteneurs aménagés en maisons pour Haïti. Ces types de projets pour Haïti ont été nombreux, on peut citer aussi SEED, de l'Université de Clemson (Caroline du Sud). Les reconstructions après les catastrophes sont ainsi des laboratoires de l'urbanisme institutionnel.

6. Rappelons qu'il s'agit dans ce cas de cité, abstraite, de constructions politiques.

7. C'est le cas aujourd'hui de l'association Halem et des mouvements en faveur de l'habitat choisi. 


\section{RÉEEREENCES}

Boltanski, L. \& Chiapello E. 1999 Le Nouvel esprit du capitalisme. Paris : Gallimard.

Bonillo, J. -L. \& al. 1992 Marseille ville et port. Marseille: Parenthèses.

Bouillon, F. 2009 Les Mondes du squat. Paris: Le Monde, Presses Universitaire de France.

Certeau, (de) M. 1990 L'Invention du quotidien, 1. Arts de faire et 2. Habiter, cuisiner. Édition établie et présentée par L. Giard. Paris: Gallimard (Folio essais).

Comité des Transports Maritimes de l'OCDE, 2003 La Sécurité dans les transports maritimes. Facteurs de risques et répercussions économiques. Paris: OCDE (Rapport).

Dufournet, P. 1979 Hector Horeau précurseur. Idées techniques Architecture. Paris: Charles Massin.

Fiore, C. 1982 Production de la circulation. Paris: EHESS (Thèse de sciences économiques).

Graham, S. \& Marvin, S. 2001 Splintering Urbanism. Networked infrastructures, technological mobilities and the urban condition. Londres: Routledge.

Horeau, H. 1868 Assainissement, embellissement de Paris. Édilité Urbaine mise à la portée de tout le monde. Paris: G.A.B.

Huizinga, J. 1938 Homo Ludens. Essai sur la fonction sociale du jeu. Paris: Gallimard.

Karpik, L. 2007 L'Économie des singularités. Paris: Gallimard.

Kopp, A. 1978 Ville et Révolution. Architecture et urbanisme soviétiques des années vingt. Paris: Anthropos.

Krugman, P 1998 L'Économie auto-organisatrice. Paris: Bruxelles-De Boeck.

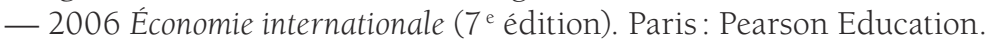

Mantoux, P. 1906 La Révolution industrielle au XVIII ${ }^{e}$ siècle; essai sur les commencements de la grande industrie moderne en Angleterre. Paris: Société de librairie et d'édition.

Michallet, A. 2004 Qu'est-ce que la mondialisation? Paris: La Découverte.

Le Marchand, A. 1997 Le Syndicalisme docker au Havre depuis 1947; de l'action structurante à la double contrainte, Cahiers du Grhis, n 7.

- 2007 Travail mobile et habitat non-ordinaire dans les villes portuaires. In V. Laflamme \& al. (Dir.) Le Logement précaire en Europe. Aux marges du palais. Paris: L'Harmattan.

Leroi-Gourhan, A. 1971 L'Homme et la matière. Paris: Albin Michel

Polanyi, K. 1983 La Grande transformation: aux origines politiques et économiques de notre temps [trad. C. Malamoud \& M. Angeno]. Paris: Gallimard (Bibliothèque des sciences humaines).

Raveneau, G. \& Sirost, O. 2001 Le Camping ou la meilleure des républiques, Ethnologie française 4/2001 (Vol. 31), 669: 680 .

Sirost, O. 2001 Camper ou l'expérience de la vie précaire au grand air, Ethnologie française 4/2001 (Vol. 31), $581: 589$.

Wallis, A. 1997 Wheel Estate. The Rise and Decline of Mobil-Homes. Baltimore: John Hopkins University Press. 


\section{RÉSUMÉ}

Les Cités en conteneur ou l'habitat dans la logistique globale. Le conteneur en tant qu'habitation fut d'abord le produit d'un détournement de la part de salariés et de travailleurs non intégrés à la « grande entreprise ». À l'origine, cet habitat n'est pas produit par les acteurs et entreprises économiques insérés dans le commerce international. Son utilisation indique pourtant que des espaces, même apparemment marginaux, sont connectés à la circulation des marchandises. Il conquiert, ensuite, une place culturellement légitimée, à travers des projets de Cités logements pour étudiants ou de centres culturels. Ce changement d'usage s'accompagne d'un écart par rapport aux usages standard des conteneurs qui, bien que modifiés pour l'habitat conservent leurs qualités mobiles. La flexibilité de ces dispositifs incite à poser la question de l'encastrement de l'habitat dans l'économie globale et à revenir sur l'histoire de la « conteneurisation». Nous examinerons les débats accompagnant l'installation d'une cité universitaire au Havre en 2010. Nous montrerons que la thèse polanyienne du désencastrement de l'économie ne semble pas convenir pour décrire ces évolutions, car il s'agit de détournements et de ruses menant à l'innovation et non d'adaptations mécaniques aux conditions économiques. Ces innovations mettent notamment en jeu des règles juridiques et des conventions informelles sur le marché du logement. Comme moyen de conditionnement et de transport, la boîte est standard, comme moyen d'habitation, elle devient singulière, au sens de « l'économie des singularités », c'est-à-dire détachée des usages de masse.

\section{ABSTRACT}

The Cities in container or the housing in total logistics. The container was diverted at first to be of use for housing by the employees and the workers not integrated into big companies, it is not a production of the big companies and the actors inserted into the international trade. Its use indicates that all the spaces, even apparently marginal, are connected to the circulation of goods at least indirectly. This remark also costs for practices supporting speeches of breaks with the wage-earner and the consumption. It conquers a place, culturally legitimized, through housing projects of city for students or cultural centers. It keeps its mobile qualities, even if this change of custom comes along with a distance with regard to the standard. The flexibility of container housing asks the question of the embedding of the housing environment in the global economy, on the history of the container. We also examine the debates accompanying the installation of a student halls of residence in Havre in 2010. The Polanyian thesis of the disembedding of economics does not fit to describe the divertment leading to innovation. It is not just a matter of adaptation to global economic conditions. On the other hand, the fuzzy status of the mobile housing environment remains, even if these projects tend to re-legitimize it. This transformation of the container engenders a singularisation of this object. As means of conditioning and transport, the box is standard, as means of house, it becomes singular, in the sense of "the economics of singularities". These innovations involve legal rules and informal conventions.

\section{MOTS CLÉS}

Habitat, conteneur, convention, logistique, institution, détournement

\section{KEYWORDS}

Housing, container, convention, logistics, institution 